Original Article

\title{
ROTATION OF UPPER LIMBS IN THE OVERWEIGHT
}

\section{Arunachalam Kumar}

Professor of Anatomy, K.S. Hegde M edical Academy, Nitte University, M angalore - 575 018, India.

\section{Correspondence :}

\section{Arunachalam Kumar}

Director (R \& D), Nitte University, Mangalore - 575 018, India.

E-mail : DirectorRD@ nitte.edu.in

\section{Abstract:}

The human body's structure is remarkable in that it is basically uniform across the populations. Apart from a few dissimilarities in skin color and external features, at the visceral and microscopic levels, man is unusually identical. M inor ethnic variations, both acquired or congenital and atavistic traits apart, human morphology is universal.

In this brief communication I present and discuss an observation made on the position of the free upper limb in the erect adult. It is seen that body weight influences forelimb position. In the stout, stocky and overweight, the upper limb is at rest, held medially rotated (axially); the degree of axial rotation being directly related to the increase in body weight.

Keywords: limb rotation, gleno-humeral joint, obesity

\section{Introduction:}

Physical anthropology is not just the study of human structure or morphology. It is a living science too; all one has to do, is to observe the living. A recent observation of mine concerns the resting position of the upper limb in overweight and obese individuals when was one made in overweight individuals.

In 'normal weight for age' and height people, the upper limbs are held dangling down the sides of the body (in a position of rest), with the palms facing or held adjacent to the thigh. In other words, the free limb is held in a semipronated forearm positus, with the thumb facing forwards (anteriorly).

Now shift focus to the obese: the palm is held facing backwards (posteriorly), the hemi-pronated forearm now over-rides a medially rotated humerus. The thumbs, do not point anteriorly (forward) but medially (inward), the back

\section{Access this article online Quick Response Code}

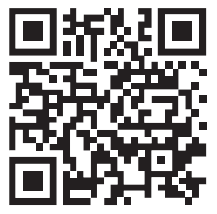

of the hand (the dorsum of the palm) face the onlooker. ${ }^{1}$ In individuals midway between slim and obese, (the stocky, overweight or stout) the upper limb is partially rotated medially. The dorsum of the palm turns more medially as the body weight increases: the degree of axial rotation being directly related to the rise in weight. The heavier one is, the more medially rotated the free upper limb is in the erect posture (see figure)
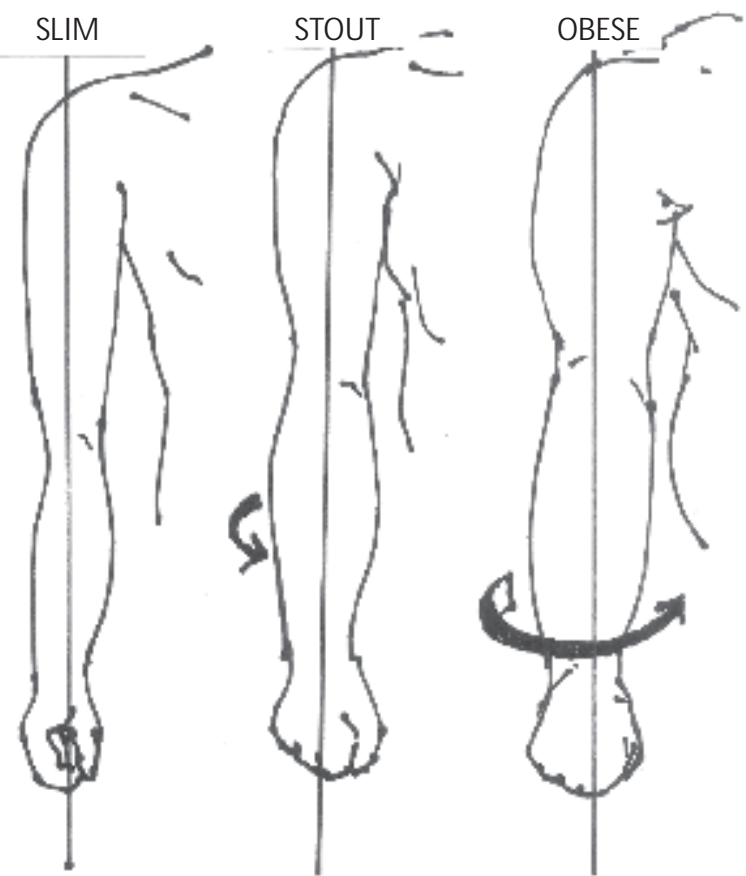

Note The alteration in the degree of axial rotation (medially) of the free upper limb in the transition from slim (thin) to overweight to obese 


\section{Discussion :}

In the obese, at rest, the forearm and forearm, as a single unit held rotated in the vertical long axis medially to the extent of $90^{\circ}$. The oddity in positioning, is seen in both sexes, but in infants, perhaps due to a difference in pattern of deposition of fat and adipose tissue, it is not apparent or observable. The body morphology induced alteration in the position of the freely suspended upper limb raises a number of questions.

How or why forelimb anatomy is altered in the overweight is a mystery. The questions now raised by this oddity are:

At what precise stage in the weight gain graph does the axial (vertical) medial rotation start?

Is the rotation reversible by reducing weight or dieting? What happens to the limb position in those yo-yoing between bulimia and anorexia?

Why does the limb change its anatomical status and position when weight is gained?

What is the relationship (if any) between the individual's adipose tissue (fat) content, lipid profile and its metabolism to the degree of axial rotation in the forelimbs?

\section{References}

1. Arunachalam Kumar (2003) The 'Thumb Sign' in the Overweight, British Medical Journal (Asian Ed.) Vol.19: No.3: pp 16-17

2. Arunachalam Kumar, Forelimb axial rotation and obesity https:// groups. yahoo.com/neo/groups/ixedoc/conversations/topics/5

3. Arunachalam Kumar (2002) The 'Thumb Sign' in obesity, Human Races Monthly, Vol.1, No.10
Does the range of axial rotation occur only at the glenohumeral joint or do the radio-ulnar joints also contribute to its degree and range?

Is it possible that in the obese, the additional adipose tissue deposited in the axilla and juxta-scapular regions, may slightly alter the anatomical position of the scapula, shifting the glenoid to face more anteriorly than just the normal antero-laterally? Any displacement in glenoid angularity, even minuscule, could significantly alter the insitu position of the suspended free upper limb, rotating it medially - apparently and actually (assuming that the rotation is initiated and maintained only at the shoulder joint).

M ore interestingly, could one apply the range of rotation as a 'rough' and index for estimating or assessing body weight status?

The human form holds an amazing maze of cryptic, and as yet undiscovered bio-mechanisms. That the in-situ rotation dynamics of the upper limb along it's vertical $\mathrm{axis}^{2,3}$, can be used as a kinetically quantifiable external indicator to body weight status is quite an interesting proposition. 